\title{
Programa de entrenamiento en potencial de aprendizaje para niños colombianos con dificultades de aprendizaje en Matemáticas
}

\author{
Dynamic assessment program for Colombian children \\ with learning disabilities in Mathematics (LDM)
}

Alejandro Sánchez-Acero ${ }^{1}$ y María Belén García-Martín ${ }^{2}$

\begin{abstract}
${ }^{1}$ Máster en Enseñanza de las Ciencias Exactas y Naturales. Licenciado en Matemáticas. Especialista en Pedagogía y Didáctica de las Matemáticas. Doctorando en Psicología. E-mail: franciscoa.sancheza@konradlorenz.edu.co

${ }_{2}^{2}$ Doctora en Psicología. Directora de la Especialización en Evaluación Clínica y Tratamiento de Trastornos Emocionales y Afectivos y de la Maestría en Psicología Clínica. Investigadora Principal del Centro de Investigaciones de Psicología CIP. Directora del Grupo de Investigación en Ciencias del Comportamiento. E-mail: maria.garcia27@unisabana.edu.co
\end{abstract}

\section{Fundación Universitaria Konrad Lorenz. \\ Bogotá, Colombia.}

\section{Resumen}

Las dificultades de aprendizaje en Matemáticas (DAM) han sido estudiadas por parte de los investigadores en los últimos años. La literatura sobre su clasificación y su identificación en niños ha sido relevante; sin embargo, es poca la bibliografía actual acerca de cómo superar dichas dificultades en niños. El presente estudio tuvo como objetivo la validación de un protocolo para mejorar habilidades en el campo de las matemáticas. La muestra se componía de 59 niños con esta característica, además del bajo rendimiento en dicha área. Los participantes tenían entre 7 y 10 años y estaban cursando entre segundo y cuarto año de primaria. El diseño es cuasiexperimental con medidas pretest-postest y grupo control en lista de espera. El programa constó de cinco semanas, con una sesión semanal de forma grupal con una duración de 50 minutos aproximadamente. Los instrumentos de evaluación fueron: TEDI-MATH para la identificación de las DAM y tres subpruebas de la WISC-IV, dígitos, conceptos y claves. Los resultados de la investigación arrojaron diferencias significativas entre el grupo control y experimental para todas las pruebas de la TEDI-MATH y para la subprueba de conceptos en WISC-IV. Los resultados son coherentes con otras investigaciones relacionadas con la comorbilidad entre los campos del lenguaje y las matemáticas. Se concluye que, al entrenar aspectos relacionados con la adquisición del lenguaje mejora la comprensión y el manejo de las competencias en matemáticas en los niños. Palabras clave: DAM, potencial de aprendizaje, escolares, matemáticas, programa de entrenamiento

Abstract
Learning Disabilities Mathematical LDM,
have been the subject of study since the
decade of the 70 `s, advances in this field have
allowed a correct identification and classifica-


tion through the design of various instruments for this objective. The number of investigations that address intervention protocols to improve this type of difficulties in children is even more limited. On this way, comorbidities have also been found different researches with the Language Learning Disabilities LLD, and as well as their relationship with the neurological development of children. It is estimated that in countries like Germany, USA, and Spain, the percentage of children with LDM is between 3\% and $8 \%$. In particular, in Colombia at the moment, there is very little research that allows identifying these difficulties in children, as well as very little research on the different types of intervention to improve these difficulties in Math. In General, there are little current literature on how to overcome such difficulties in children. An intervention based on the perspective of dynamic assessment would be useful for children who have these difficulties. The dynamic assessment implies two important concepts: activity and modifiability. The tester and the inquired assume an active role, where the tester intervenes and modifies the interaction with the inquired with the purpose of successfully inducing learning. The inquired is directed and reinforced to assume an active role in searching and organizing information. The product of the assessment is modifiability or change in cognitive functioning. This study presents the validation of a protocol based on dynamic assessment to improve skills in the field of mathematics. The sample was 59 Colombian children from public schools in the city of Bogotá. Participants were between 7 to 10 years old and from second to fourth grade. They had Mathematics Learning Difficulties in evaluated with TEDI-MATH on short version. They were distributed in 28 boys and 31 girls with this characteristic. And children who were undergoing psychological, medicated and/or clinical treatment processes were excluded. The design used was quasi-experimental with pretest-posttest measures and control group on the waiting list. The program considered five weeks, with one weekly session of group with a duration of approximately 50 minutes. The evaluation instruments were: TEDI-MATH which was used for the identification of the MLD; $3 \mathrm{sub}$ tests of the WISC-IV, digits, concepts and keys. And PAMATH-C protocol was used for the intervention with children with the aforementioned difficulties. The results of the investigation showed significant differences between the control and experimental group for all the TEDI-MATH tests and for the subtest of concepts in WISC-IV. The results are consistent with other research related to comorbidity between the fields of language and mathematics. The results showed that like other research based interventions with Learning Potential training programs are effective in improving the different metacognitive abilities among the participants. and has a significant effect on the decrease in Mathematical Learning Disabilities. This research leaves projections for an application to a larger population in Colombia and in South America of the TEDYMATH test on short version, since good discrimination was observed for children with LDM. The application of the PAMATH-C protocol in other types of rural and urban population throughout the territories will improve the identification of children with difficulties. In addition, the contribution for the design of new tests based on dynamic evaluation and its form of application to improve other types of difficulties in both children and adults. Therefore, an immense methodological field to develop future protocols based on the Learning Potential. It is concluded that when training aspects related to language acquisition, it improves the understanding and management of mathematics in children.

Keywords: LDM, learning potential, scholars, mathematics, training program 


\section{Introducción}

\section{Dificultades de aprendizaje en Matemáticas}

Las dificultades de aprendizaje en Matemáticas (DAM) han sido estudiadas durante los últimos 50 años y se han empleado diferentes denominaciones para este tipo de dificultad. Geary (1993) en sus primeras investigaciones utilizó el término discapacidad matemática [mathematical disability] [MD], posteriormente Geary y Hoard (2001) lo llamaron discapacidad de aprendizaje aritmética [arithmetic learning disability] [AL, ALD], y en sus últimas investigaciones, Geary (2011) lo ha generalizado como discapacidades en el aprendizaje matemático [mathematical learning disabilities] (MLD). Cada término que utilizó este autor permitió profundizar y especificar mucho más la complejidad de la concep- ción de las DAM. En este sentido, se puede mencionar que el término discalculia es investigado por Butterworth (2005) y empleado en el Manual Diagnóstico y Estadístico de Trastornos Mentales DSM-V (American Psychiatric Association, APA, 2013) y se enmarca en el grupo de los trastornos específicos del aprendizaje con inicio en la infancia, la niñez o la adolescencia. El manual hace referencia a la discalculia como el trastorno en el cálculo.

Jiménez (2016) menciona tres características principales de las DAM: concepto de números, operaciones matemáticas básicas y solución de problemas. Las clasificaciones existentes sobre las DAM han permitido diseñar diferentes instrumentos de evaluación para su correcta identificación. A continuación, se describen algunas pruebas mencionadas en la mayoría de las investigaciones que permiten evaluar las DAM.

Tabla 1.

Pruebas para la identificación de las DAM en español y en inglés.

\begin{tabular}{|c|c|c|}
\hline & \multicolumn{2}{|c|}{ Pruebas que evalúan dificultades en Matemáticas } \\
\hline \multirow{3}{*}{ 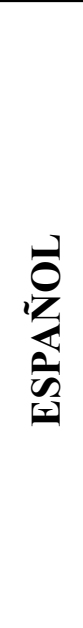 } & $\begin{array}{c}\text { Prueba de aptitud y rendimiento } \\
\text { matemático PCM (Olea, Ahumada y } \\
\text { Líbano, 1993). }\end{array}$ & $\begin{array}{l}\text { Nociones previas } \\
\text { Simbolización } \\
\text { Disposición/Cálculo }\end{array}$ \\
\hline & $\begin{array}{l}\text { Prueba Evolutivo-Curricular de Mate- } \\
\text { máticas de Tordesillas (PRECUMAT) } \\
\text { (Blanco, 1999). }\end{array}$ & $\begin{array}{l}\text { Numeración escrita, conteo, cálculo, sentido } \\
\text { del número, problemas verbales, hechos } \\
\text { numéricos y relaciones conceptuales y estima- } \\
\text { ción. }\end{array}$ \\
\hline & $\begin{array}{l}\text { TEDI-MAT por Grégoire, Noël y } \\
\text { Van-Nieuwenhoven (2005) }\end{array}$ & $\begin{array}{c}\text { Está compuesta por seis subpruebas: } \\
\text { contar, numerar, comprensión del sistema } \\
\text { numérico, sistema en base 10, operaciones } \\
\text { lógicas, operaciones y estimación del tamaño. }\end{array}$ \\
\hline
\end{tabular}




\begin{tabular}{|c|c|c|}
\hline & $\begin{array}{c}\text { TEMA-2 Test of Eraly Mathematics } \\
\text { Ability (Ginsburg y Baroody, 1990) }\end{array}$ & $\begin{array}{c}\text { Conteo, numeración, magnitud relativa, cálculo, } \\
\text { lectura y escritura de números, resolución de } \\
\text { problemas y sistema numérico decimal. }\end{array}$ \\
\cline { 2 - 4 } & $\begin{array}{c}\text { Key- Math Diagnostic Arithmetic Test } \\
\text { (Connolly, 1998). }\end{array}$ & $\begin{array}{c}\text { Numeración, números racionales, geometría, } \\
\text { operaciones aritméticas, cálculo, medida, tiempo } \\
\text { y dinero, estimación, interpretación de datos y } \\
\text { resolución de problemas. }\end{array}$ \\
\cline { 2 - 4 } & $\begin{array}{c}\text { Software' Dyscalculia Screener de Butter- } \\
\text { worth (2005). }\end{array}$ & $\begin{array}{c}\text { Tiempo de respuesta, numeración, compara- } \\
\text { ción de números y operaciones aritméticas. }\end{array}$ \\
\hline
\end{tabular}

Fuente: Elaboración propia.

\section{Estudios clínicos sobre intervenciones en las DAM}

Estudios sobre las DAM indican que entre el $1 \%$ y el $8 \%$ de los niños en etapa escolar presentan este tipo de dificultades (Geary, 1993, 2004; Jáuregui, Mora-Ruiz, Oviedo-Mosquera, Pabón-Rodríguez y Rodríguez-Osorio, 2016). Además, un $25 \%$ de estos niños presentan algún tipo de dificultad específica de aprendizaje (DEA) (Romero y Lavigne, 2004, 2011) y un $55 \%$ tiene dificultades relacionadas con la lectura y la escritura.

Concretamente, sobre la prevalencia de las DAM se encuentran diferentes estudios que arrojan valores entre el $1 \%$ y $10 \%$ en niños en etapas escolares. La variación entre los porcentajes de prevalencia se da primordialmente por factores relacionados con la edad, los factores de inclusión y exclusión, el idioma $\mathrm{y}$, en general, por el contexto sociocultural (Aguilar, Aragón y Navarro, 2015; Gross-Tsur,Manor y Shalev,1996).

Existen algunos supuestos cognitivos que llevan a identificar las causas de las DAM en los niños, entre ellos se habla de factores de dominio general como la memoria de trabajo, la inteligencia y el lenguaje, y de dominio específico como problemas con el sentido numérico, el sistema de precisión-exactitud numérica (SPN), el sistema de aproximación numérica (SAN), o el acceso al sentido numé- rico (Butterworth, 2005). Este tipo de dominios son los que usualmente han sido claves para el diseño de diferentes programas de entrenamiento (Moll, Göbel, Gooch, Landerl, Karin y Snowling, 2014).

Moreno y Daza (2014) implementaron la enseñanza de estrategias metacognitivas para el desarrollo de habilidades de resolución de problemas matemáticos en tres estudiantes de $7^{\circ}$ año de bachillerato de un colegio de Bogotá, Colombia. Se recogieron medidas pretest-postest. El programa constó de ocho sesiones de 40 minutos cada una, en donde se enseñaron diferentes estrategias metacognitivas para la resolución de una serie estandarizada de problemas matemáticos. Los resultados mostraron que tras la enseñanza de las estrategias, los estudiantes desarrollaron habilidades de planeación y supervisión regular del proceso de resolución de problemas, conciencia de las habilidades y autorregulación, evidenciándose un logro en el desempeño de las actividades propuestas.

Zerafa (2015) analiza el efecto de un programa de intervención a dos niños de 10 años y uno de 7 años con DAM, que fueron seleccionados tras realizar la evaluación a 15 niños precandidatos al estudio, a través del Dyscalculia Screener. Posteriormente, realiza una evaluación pretest (Catch up Numeracy) para establecer las debilidades y fortalezas que tiene la muestra seleccionada, en torno a los componentes numéricos. Los componentes 
de las actividades de la intervención (Catch up numeracy programme) fueron en torno al entrenamiento en habilidades numéricas, las cuales se enseñaron según el resultado preliminar de la evaluación. En el postest (Catch up Numeracy) los niños mostraron mejoría en los componentes numéricos del test. Igualmente, lograban hacer sumas y restas mentalmente, sin necesidad de acudir a los dedos, como lo hacían en el pretest. El estudio concluyó que los niños mostraron mayor interés por las matemáticas, así como una mayor capacidad metacognitiva para reflexionar sobre lo que habían aprendido, en contraste con sus conocimientos previos.

Mutlu y Akgün (2017) implementaron un programa de intervención basado en un material instruccional asistido por un computador, dirigido a dos niños y una niña que cursaban tercer año de primaria en diferentes escuelas y que presentaban DAM. Como estrategias de evaluación pretest/postest, los autores utilizaron el PANAMATH (Halberda, Mazzocco y Feigenson, 2008), el cual utiliza tareas de comparación de puntos, y el subtest de línea numérica del Dyscalculia Screening Tool (Olkun, Altun, Cangöz, Gelbal y Sucuoğlu, 2012). La intervención duró seis semanas: se realizaron cinco sesiones semanales de 20-30 minutos de carácter individual, a través del material virtual. Se encontró que los niños presentaban dificultades para hacer sumas de uno y dos dígitos y responder preguntas en torno al valor posicional de los números. Por tanto, se brindó entrenamiento en estas tareas, así como en el desarrollo de otras habilidades matemáticas. Los resultados arrojaron un aumento en la agudeza del sentido numérico de los niños, que también se evidenció en una mayor rapidez de respuesta en los tests de comparación de puntos y conteo numérico, logrando una mejoría en las habilidades numéricas.

\section{El enfoque del aprendizaje mediado en las intervenciones en DAM}

Siguiendo los fundamentos de una aproximación conductual, se considera que los errores escolares que un niño puede cometer, no son permanentes, sino que pueden ser modificados a través de la aplicación apropiada de los principios del aprendizaje.

Por otro lado, el modelo de modificabilidad cognitiva (Feuerstein, 1990a, 1990b, 1990c, 1996; Feuerstein y Rand, 1974) y su idea de la experiencia de aprendizaje mediado, especifica que al individuo se le proporcionan instrumentos de adaptación y aprendizaje con el fin de hacer a la persona capaz de utilizar eficientemente la exposición directa al aprendizaje. En este modelo se hace referencia al concepto de potencial de aprendizaje que todo individuo presenta, definiéndolo así como la capacidad general que tiene un sujeto para sacar provecho de un entrenamiento dirigido sobre la enseñanza en resolución de problemas más o menos complejos en los que en un principio el individuo fracasa inicialmente. (Calero y Márquez, 1998).

Esta idea sigue siendo considerada y ha sido reformulada por Fernández-Ballesteros, Espinosa, Colom y Calero (1997) cuando hablan de la mediación contextual. El contexto también se incluye en los procesos cognitivos, no porque las demandas del ambiente requieran ciertas habilidades (y/o procesos), sino porque el contexto soporta todo el proceso de socialización con el que la sociedad, a través de la familia, la escuela, etcétera, media en el aprendizaje. Se puede decir entonces que se asume que las habilidades intelectuales y el aprendizaje son producto de la interacción entre la persona y el contexto en el que vive, consideraciones que coinciden con el modelo conductual.

Las características mencionadas refieren que el aprendizaje mediado es un modelo de evaluación e intervención que implica un proceso que los integra, es decir, que la aplicación de una situación de prueba no implica sólo obtener información sobre el nivel cognitivo actual del individuo, sino que en el momento de evaluarlo se consideran los errores y los aciertos y en ese momento se identifican las estrategias a seguir para 
someterlo a un entrenamiento y aprovechar su potencial de aprendizaje relacionado con los procesos cognitivos apropiados a la tarea (Lauchlan y Elliot, 2001; Sternberg y Grigoreko, 2001).

El aprendizaje mediado se refiere a una experiencia de aprendizaje en la que otra persona da apoyo (un padre, un maestro, un terapeuta) se interpone entre el organismo (el niño) y el entorno, influyendo intencionalmente la naturaleza de la interacción. Esto se conoce como un modelo de evaluación dinámica, diseñado para evaluar el potencial del niño o adolescente, con el fin de alcanzar un crecimiento en los procesos cognitivos específicos, primeramente, a través de la exposición guiada de problemas y procesos de pensamiento y. después, en forma independiente (Calero, Navarro, Armedo, García-Berben y Robles, 2000; Feuerstein, 1984; Robles y Calero, 2003; Wiedl, Wienöbst y Schöttke, 2000).

En Latinoamérica, se puede observar que las intervenciones para mejorar las DAM en niños, son escasas y no existen estudios con intervenciones en potencial de aprendizaje como herramienta que permita mejorar estas dificultades. Es por ello que el presente estudio pretende probar el efecto de un entrenamiento en potencial de aprendizaje para mejorar las DAM en niños colombianos.

\section{Método}

\section{Participantes}

Los participantes fueron 59 niños colombianos de escuelas públicas de la ciudad de Bogotá, distribuidos en 28 niños (47.5\%) y 31 niñas $(52.5 \%)$; con edades entre 7 y 10 años $(M=8.24 ; D=.817)$, divididos en dos grupos: uno experimental con 28 niños (47.5 $\%)$ y otro control con 31 niños $(52.5 \%)$. Los participantes están entre segundo y cuarto año de primaria. Presentaban dificultades de aprendizaje en matemáticas evaluados con TEDI-MATH, versión corta (Sánchez-Acero y García-Martín, 2019a). Se excluyeron niños que estuvieran en procesos psicológicos, medicados y/o tratamientos clínicos.

Tabla 2.

Distribución por edad de los participantes por grupos experimental y control.

\begin{tabular}{|c|c|c|c|}
\hline Edad & Grupo Experimental \% & Grupo Control \% & Total \% \\
\hline $\mathbf{7}$ & 15.3 & 51.0 & 20.3 \\
\hline $\mathbf{8}$ & 13.6 & 25.4 & 39.0 \\
\hline $\mathbf{9}$ & 16.9 & 20.3 & 37.3 \\
\hline $\mathbf{1 0}$ & 1.7 & 1.7 & 3.4 \\
\hline Total general & 47.46 & 52.54 & 100.00 \\
\hline
\end{tabular}

\section{Diseño y procedimiento}

Se trató de un estudio cuasiexperimental pretest-postest con grupo control en lista de espera, el cual recibiría la intervención una vez que culminará la intervención al grupo experimental. fases:
1. Se envió una carta a los rectores de los centros educativos con una explicación del proyecto y la solicitud de colaboración.

2. Con aquellos que aceptaron colaborar, se realizó una entrevista con el fin de presentar el proyecto y entregar los consentimientos y asentimientos informados para los padres de familia de los participantes del estudio. 
3. Tras la recepción de los consentimientos, se llevó a cabo la aplicación de la prueba TEDI-MATH en su versión corta y WISC-IV de forma individual iniciando con los niños de segundo grado hasta culminar con niños de cuarto grado. 4. Se determinaron los niños que tenían puntajes menores al $40 \%$ en dos o más subpruebas aplicadas de la TEDI-MATH (Sánchez-Acero y García-Martín, 2019a). 5. Se realizó una selección aleatoria de los participantes para determinar el grupo al cual iban a pertenecer (control o experimental) mediante el uso de Excel y su asignación mediante la función RAMDOM (1: experimental, 2: control)

6. Se aplicó el protocolo de intervención en potencial de aprendizaje diseñado en cinco sesiones al grupo experimental. Se organizaron seis grupos de cinco participantes organizados por su grado de escolaridad.

7. Se aplicó de nuevo la prueba TEDIMATH y las pruebas de inteligencia a los dos grupos, control y experimental.

8. Finalmente, se envió a cada padre de familia un informe con los resultados encontrados tras el entrenamiento y un informe general al colegio.

\section{Instrumentos}

Los instrumentos de evaluación e intervención utilizados fueron:

\section{Test para el Diagnóstico de las Competencias Básicas en Matemáticas [TEDI-MATH] (Grégoire, Noël y Van- Nieuwenhoven, 2005)}

Este test evalúa las competencias básicas matemáticas de las siguientes áreas: a) Contar; b) Numerar; c) Comprensión del sistema numérico; d) Operaciones lógicas; e) Operaciones y f) Estimación del tamaño. La prueba determina 12 factores generales, entre los seis subtests, obteniendo así 12 puntuaciones para toda la prueba.

En este estudio se evaluaron únicamente las áreas que parecen identificar con fiabilidad dificultades de aprendizaje en matemáticas en niños colombianos (Sánchez-Acero y GarcíaMartín, 2019a), que son:

- Operaciones con enunciado aritmético que determina tareas como sumas simples, sumas con huecos, restas simples, restas con huecos, multiplicaciones simples, operaciones con enunciado verbal.

- Conocimientos conceptuales enmarcados en la dimensión de operaciones y que permiten observar tareas como determinación de algoritmos básicos para operar.

- Estimación del tamaño, que presenta tareas como la comparación de modelos de puntos dispersos y tamaño relativo.

El análisis de fiabilidad del instrumento (a través del $\alpha$ de Cronbach) ha mostrado valores adecuados para cada una de sus dimensiones, con puntuaciones entre .84 y .99 .

\section{Test de Inteligencia Wechsler para niños [WISC-IV] (Wechsler, Kaplan, Fein, Kramer, Morris, Delis y Maerlender, 2004)}

Se trata de un test que evalúa inteligencia general en niños desde los 6 a los 16 años. Este test contiene 10 subtests agrupados en cuatro áreas: comprensión verbal, razonamiento perceptivo, memoria de trabajo y velocidad de procesamiento. Para el presente estudio se aplicaron las subpruebas de: a) Comprensión verbal (CV) utilizando el subtest Conceptos; b) Memoria de Trabajo (MT) usando el subtest de Dígitos y c) Velocidad de Procesamiento (VP) con Claves. La fiabilidad de esta prueba oscila entre .75 y .91 entre sus factores.

\section{Programa de Potencial de Aprendizaje para el entrenamiento en DAM [PAMATH-C] (Sánchez-Acero y García-Martín, 2019b) ${ }^{1}$}

Consta de cuatro sesiones que se realizan de forma grupal. La periodicidad es una

${ }^{1}$ El link del protocolo completo se encuentra en: https://cutt.ly/pyVhXTE 
sesión semanal con una duración aproximada de una hora. A partir de distintas estrategias de mediación, el protocolo aborda las siguientes variables de estudio:

- Decisión numérica: comprensión verbal y razonamiento verbal. Identificación del número en contexto. Ejemplo: "esta mañana desayuné (2) dos huevos y (1) un pan". ¿Puedes decirme cuáles palabras en la oración anterior son números?

- Decisión numérica oral: comprensión y razonamiento verbales. Ejemplo: "Te voy a decir unas palabras y debes decirme si estas son números o no".

- Juicio gramatical: comprensión verbal y razonamiento verbal. Ejemplo: "Un amigo me ha dicho que tiene ... canicas". ¿Puedes decirme cuándo está bien dicho? - Comparación de números orales: comprensión verbal y razonamiento abstracto. Ejemplo: "Te voy a decir unos números y debes decirme cuál es más grande".

- Operaciones con enunciado aritmético: razonamiento numérico-matemático. Ejemplo: Se presentan láminas con operaciones $6+8=\ldots, 2+2=\ldots$ sin leerlas.

- Operaciones con enunciado verbal: comprensión verbal y razonamiento numérico-matemático. Ejemplo: "Luis tiene 2 canicas y gana otras 2 , ¿cuántas tendrá en total?"

- Conocimientos conceptuales: Razonamiento matemático. Solución de problemas. Ejemplo: se presentan en ejercicios las propiedades conmutativas, definición de multiplicación, etcétera. Si se conoce que $2+2+2=6$, ¿se podrá conocer cuál es resultado de $2 \times 3=\ldots$ ?

- Comparación de modelo de puntos: Razonamiento matemático. Ejemplo: se presentan puntos y se debe indicar dónde existe mayor cantidad.

- Tamaño relativo: Solución de problemas. Ejemplo: Se presentan tres números, el dato el intermedio se toma como referencia y se le pregunta al niño sobre cuál es el número más cercano en valor con relación dato de referencia. Él debe señalar si es el de la derecha o el de la izquierda.

El desarrollo de estas habilidades es progresivo en cada sesión, lo que implica que se va incrementando el nivel de dificultad mediante el uso de números con mayor cantidad y la complejidad en el vocabulario y léxico utilizado en las situaciones.

Las actividades que se desarrollan sesión a sesión son:

Sesión 1: Identificando palabras números; palabras - números a contexto; cruci número para identificación de palabras - números; identificación de cantidad o valor numérico (mayor - menor) y uso funcional de los números e identificación de cantidad a partir de la medición de objetos.

Sesión 2: Comparación de números arábigos; organización de series de números arábigos; nociones de mayor que, menor que, más que, menos que, y las reglas en las matemáticas.

Sesión 3: Comparación de números compuestos; organización de números compuestos; nociones de mayor que, menor que, más que, menos que con números compuestos; las reglas en las matemáticas, y cruci-número para identificar palabras - números compuestos.

Sesión 4: Identificación de números compuestos mayores a 50; organización de series de números compuestos por tamaño, nociones de mayor que, menor que, más que, menos que con números compuestos y las reglas en las matemáticas.

Por ejemplo, una sesión cuyo objetivo es la de entrenar la comparación de números en un contexto se inicia con preguntas como ¿Tú tienes hermanos? ¿Cuántos hermanos tienes? ¿Eres el mayor? ¿Eres el menor? ¿Cuántos años tienen tus hermanos? La mediación se daría mediante el diálogo con los niños sobre 
las edades; por ejemplo, se les pregunta sobre cuántos años más tiene uno que otro. Se guían las respuestas y se refuerza la conducta de conteo hacia adelante y hacia atrás para facilitar su respuesta. Se le solicita indicar el nombre de cada uno de los hermanos al frente de cada cara y la edad mediante una figura dada en la cual relacionan los hermanos con sus respectivas edades. Luego, se da el siguiente ejemplo a los niños: Pedro tiene 2 años, Yo (nombre del niño) tiene 8 años y Juan tiene 17 años. ¿Quién es menor? (varían los nombres dependiendo de lo que coloque el niño). Pedro o Juan, Juan o tú, tú o Pedro. ¿Quién es mayor? Pedro o Juan, Juan o tú, tú o Pedro. El protocolo está detallado por los autores en su publicación, indicando por cada sesión los objetivos, las tareas y las competencias que desarrolla.

\section{Resultados}

Tabla 3.

Estadísticos descriptivos y prueba $t$ de Student para muestras independientes en puntuaciones pretest y postest de ambos grupos.

\begin{tabular}{|c|c|c|c|c|c|c|c|c|}
\hline Variable & & Grupo & $\mathbf{N}$ & Media & d.t & RANGO & $\mathbf{T}$ & $\mathbf{p}$ \\
\hline \multirow{4}{*}{$\begin{array}{l}\text { Sistema numé- } \\
\text { rico oral }\end{array}$} & \multirow{2}{*}{ Pretest } & Experimental & 28 & 22.00 & 2.611 & \multirow{4}{*}{$0-45$} & \multirow{2}{*}{.186} & \multirow{2}{*}{.853} \\
\hline & & Control & 31 & 21.84 & 3.984 & & & \\
\hline & \multirow{2}{*}{ Postest } & Experimental & 28 & 24.96 & 2.365 & & \multirow{2}{*}{2.840} & \multirow{2}{*}{.007} \\
\hline & & Control & 31 & 22.58 & 3.956 & & & \\
\hline \multirow{4}{*}{$\begin{array}{l}\text { Operaciones } \\
\text { con enunciado } \\
\text { aritmético }\end{array}$} & \multirow{2}{*}{ Pretest } & Experimental & 28 & 27.07 & 10.349 & \multirow{4}{*}{$0-54$} & \multirow{2}{*}{.327} & \multirow{2}{*}{.745} \\
\hline & & Control & 31 & 26.26 & 8.741 & & & \\
\hline & \multirow{2}{*}{ Postest } & Experimental & 28 & 37.36 & 12.145 & & \multirow{2}{*}{2.478} & \multirow{2}{*}{.017} \\
\hline & & Control & 31 & 30.45 & 8.805 & & & \\
\hline \multirow{4}{*}{$\begin{array}{c}\text { Operaciones } \\
\text { con enunciado } \\
\text { verbal }\end{array}$} & \multirow{2}{*}{ Pretest } & Experimental & 28 & 6.64 & 2.094 & \multirow{4}{*}{$0-12$} & \multirow{2}{*}{.707} & \multirow{2}{*}{.482} \\
\hline & & Control & 31 & 6.23 & 2.404 & & & \\
\hline & \multirow{2}{*}{ Postest } & Experimental & 28 & 8.93 & 1.654 & & \multirow{2}{*}{4.824} & \multirow{2}{*}{$<.001$} \\
\hline & & Control & 31 & 6.52 & 2.174 & & & \\
\hline \multirow{4}{*}{$\begin{array}{l}\text { Conocimientos } \\
\text { conceptuales }\end{array}$} & \multirow{2}{*}{ Pretest } & Experimental & 28 & 3.25 & 1.430 & \multirow{4}{*}{$0-8$} & \multirow{2}{*}{.362} & \multirow{2}{*}{.718} \\
\hline & & Control & 31 & 3.10 & 1.777 & & & \\
\hline & \multirow{2}{*}{ Postest } & Experimental & 28 & 4.32 & 1.634 & & \multirow{2}{*}{1.763} & \multirow{2}{*}{.083} \\
\hline & & Control & 31 & 3.61 & 1.453 & & & \\
\hline
\end{tabular}

La Tabla 3 muestra los estadísticos descriptivos de las puntuaciones pretest y postest de ambos grupos, evidenciándose las puntuaciones medias obtenidas respecto a las variables evaluadas en ambos momentos. La prueba $t$ para muestras independientes analizó las diferencias entre medias en las puntuaciones pretest del grupo experimental y el control en lista de espera en todas las variables estudiadas. Los resultados no arrojaron diferencias significativas entre las puntuaciones de ambos grupos, lo que indica que se contó con grupos equivalentes en el estudio.

Con respecto al análisis del ANOVA (Tabla 4) de medidas repetidas se compararon las puntuaciones de las medidas pretest y postest de ambos grupos con el fin de identificar si se presentan diferencias significativas desde la primera medición a la segunda.

A continuación se muestran los resultados para cada variable (ver Tablas 3 y 4): 


\begin{tabular}{|c|c|c|c|c|c|c|c|c|}
\hline Variable & & Grupo & $\mathbf{N}$ & Media & d.t & RANGO & $\mathbf{T}$ & $\mathbf{p}$ \\
\hline \multirow{4}{*}{$\begin{array}{c}\text { Estimación del } \\
\text { tamaño }\end{array}$} & \multirow{2}{*}{ Pretest } & Experimental & 28 & 15.04 & 2.575 & \multirow{4}{*}{$0-18$} & \multirow{2}{*}{-1.199} & \multirow{2}{*}{.843} \\
\hline & & Control & 31 & 15.16 & 2.267 & & & \\
\hline & \multirow{2}{*}{ Postest } & Experimental & 28 & 17.00 & 1.305 & & \multirow{2}{*}{2.145} & \multirow{2}{*}{.036} \\
\hline & & Control & 31 & 16.10 & 1.850 & & & \\
\hline \multirow{4}{*}{ Dígitos } & \multirow{2}{*}{ Pretest } & Experimental & 28 & 9.89 & 1.988 & $0-16$ & \multirow{2}{*}{.231} & \multirow{2}{*}{.818} \\
\hline & & Control & 31 & 9.77 & 1.961 & & & \\
\hline & \multirow{2}{*}{ Postest } & Experimental & 28 & 11.71 & 1.802 & & \multirow{2}{*}{1.529} & \multirow{2}{*}{.132} \\
\hline & & Control & 31 & 10.94 & 2.081 & & & \\
\hline \multirow{4}{*}{ Conceptos } & \multirow{2}{*}{ Pretest } & Experimental & 13 & 10.54 & 3.307 & $0-16$ & \multirow{2}{*}{-.368} & \multirow{2}{*}{.716} \\
\hline & & Control & 12 & 11.00 & 2.923 & & & \\
\hline & \multirow{2}{*}{ Postest } & Experimental & 13 & 15.23 & 3.563 & & \multirow{2}{*}{2.137} & \multirow{2}{*}{.043} \\
\hline & & Control & 12 & 12.18 & 3.801 & & & \\
\hline \multirow{4}{*}{ Claves } & \multirow{2}{*}{ Pretest } & Experimental & 13 & 34.77 & 7.823 & $0-45$ & \multirow{2}{*}{-.373} & \multirow{2}{*}{.712} \\
\hline & & Control & 12 & 35.75 & 4.827 & & & \\
\hline & \multirow{2}{*}{ Postest } & Experimental & 13 & 43.54 & 10.437 & & \multirow{2}{*}{1.574} & \multirow{2}{*}{.129} \\
\hline & & Control & 12 & 38.25 & 5.328 & & & \\
\hline
\end{tabular}

Tabla 4.

ANOVA de medidas repetidas para las variables evaluadas

\begin{tabular}{c|ccccc} 
Origen & $\boldsymbol{g} \boldsymbol{c}$ & $\begin{array}{c}\text { Media } \\
\text { cuadrática }\end{array}$ & $\boldsymbol{F}$ & $\boldsymbol{p}$ & $\begin{array}{c}\text { Eta al cuadrado } \\
\text { parcial }\end{array}$ \\
\hline $\begin{array}{c}\text { Sistema Numérico Oral* } \\
\quad \text { GRUPO }\end{array}$ & 1 & 36.330 & 18.415 & $<.001$ & .244 \\
$\begin{array}{c}\text { Operaciones con Enunciado } \\
\text { Aritmético* GRUPO }\end{array}$ & 1 & 273.012 & 9.493 & .003 & .143 \\
$\begin{array}{c}\text { Operaciones Enunciado } \\
\text { Verbal* GRUPO }\end{array}$ & 1 & 29.288 & 23.170 & $<.001$ & .289 \\
$\begin{array}{c}\text { Conocimientos Concep- } \\
\text { tuales* GRUPO }\end{array}$ & 1 & 2.268 & 1.936 & .170 & .033 \\
$\begin{array}{c}\text { Estimación del Tamaño * } \\
\text { GRUPO }\end{array}$ & 1 & 7.786 & 4.291 & .043 & .070 \\
$\quad \begin{array}{c}\text { Dígitos* GRUPO } \\
\text { Conceptos* GRUPO }\end{array}$ & 1 & 3.206 & 1.920 & .171 & .033 \\
$\quad$ Claves* GRUPO & 1 & 40.637 & 26.076 & $<.001$ & .531 \\
\end{tabular}

Sistema Numérico Oral: existen diferencias significativas en las puntuaciones del pretest al postest entre el grupo experimental y el control en lista de espera $(p<.05)$, observándose que el grupo experimental obtiene mejorías significativas en esta variable con respecto al grupo control (ver Figura 1). Por su parte, el tamaño del efecto de la intervención es .244. 

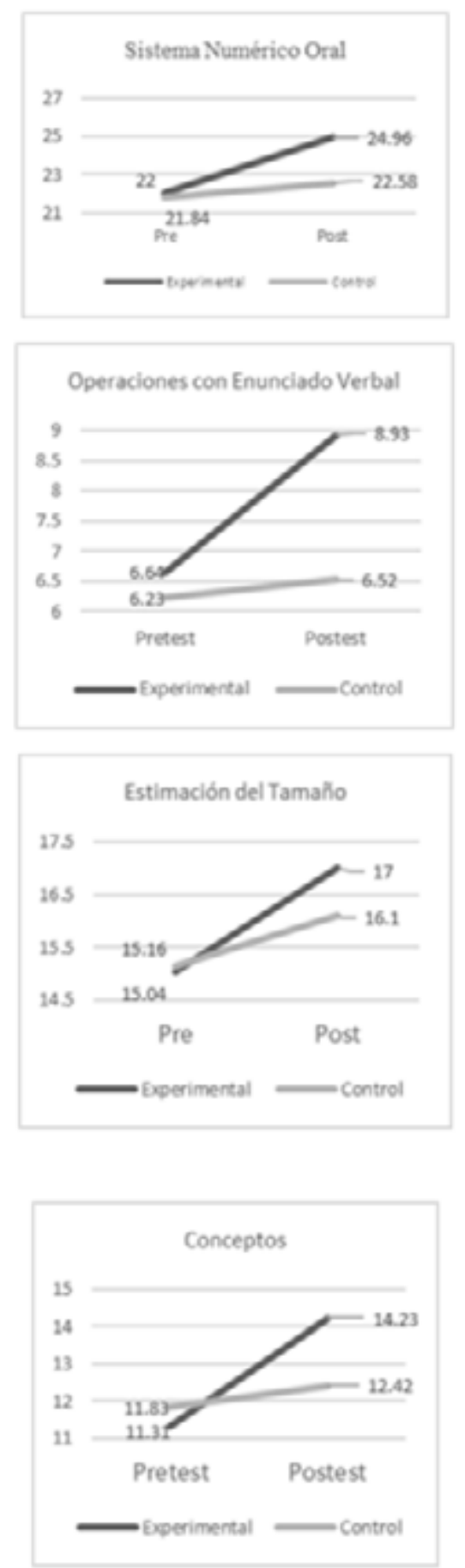

Figura 1. Valores medios en pretest y postest de las variables evaluadas.

Operaciones con Enunciado Aritmético: existen diferencias significativas en las puntuaciones del pretest al postest entre el grupo experimental y el control $(p<.05)$ con un tamaño del efecto de .143. El grupo experimental obtiene mejorías significativas con respecto al grupo control, lo cual se puede
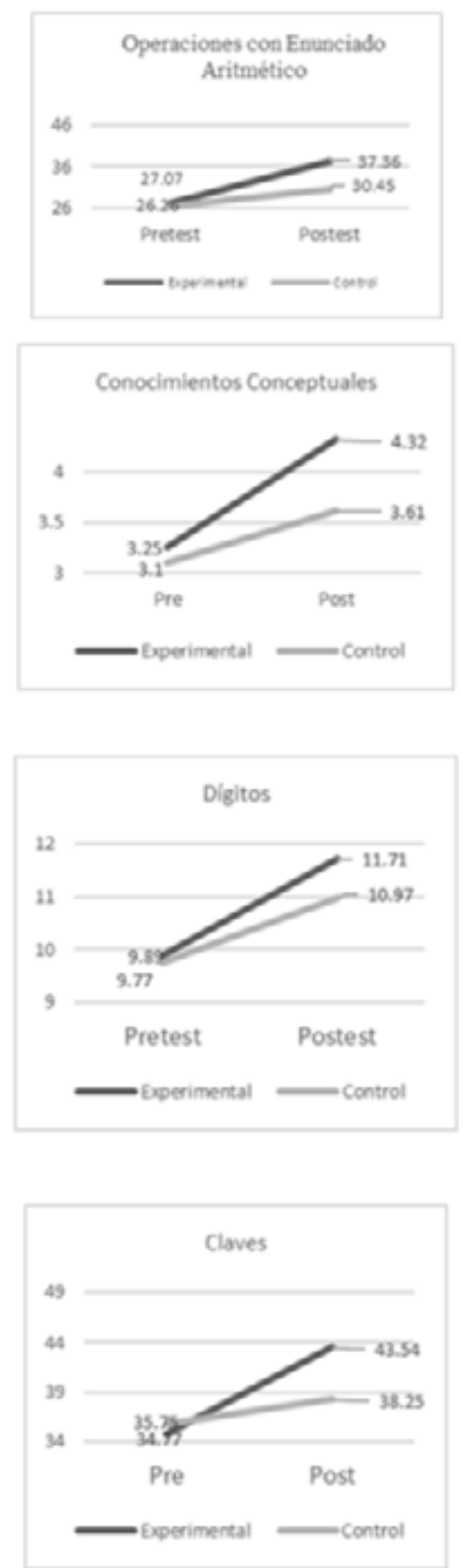

confirmar con la Figura 1.

Operaciones con Enunciado Verbal: existen diferencias significativas en las puntuaciones del pretest al postest entre el grupo experimental y el control $(p<.05)$ observándose que el grupo experimental obtiene mejorías significativas con respecto al grupo control, 
lo cual se puede confirmar con la Figura 1.El tamaño del efecto de la intervención de .289.

Conocimientos Conceptuales: no existen diferencias significativas en las puntuaciones del pretest al postest entre el grupo experimental y el control $(p=.170)$, observándose que el grupo experimental no obtiene mejorías significativas con respecto al grupo control, lo cual se puede confirmar con la Figura 1.

Estimación del Tamaño: existen diferencias significativas marginales en las puntuaciones del pretest al postest entre el grupo experimental y el control $(p<.05)$, observándose que el grupo experimental obtiene mejorías significativas con respecto al grupo control, lo cual se puede confirmar con la Figura 1.

Variable de inteligencia Memoria de Trabajo medida con Dígitos: no existen diferencias significativas en las puntuaciones del pretest al postest entre el grupo experimental y el control ( $p=.171)$, observándose que tanto el grupo experimental y el grupo control obtiene mejorías con respecto al postest, lo cual se puede confirmar con la Figura 1.

Variable de inteligencia Razonamiento Verbal medida con Conceptos: existen diferencias significativas en las puntuaciones del pretest al postest entre el grupo experimental y el control $(p<.05)$, observándose que el grupo experimental obtiene mejorías significativas en esta medida de inteligencia con respecto al grupo control, lo cual se puede confirmar con la Figura 1. Por su parte, el tamaño del efecto de la intervención en esta variable es Moderado-Alto valor de .53 .

Variable de inteligencia Velocidad de Procesamiento, medida con Claves: existen diferencias significativas en las puntuaciones del pretest al postest entre el grupo experimental y el control $(p>.05)$, observándose que el grupo experimental obtiene mejorías significativas con respecto al grupo control, lo cual se puede confirmar con la Figura 1. Por su parte, el tamaño del efecto de la intervención en esta variable es moderado alto con un valor de .449 .

\section{Discusión}

Este estudio examinó la eficacia de un protocolo de entrenamiento en potencial de aprendizaje para niños con dificultades en el aprendizaje de las matemáticas. Los resultados fueron significativos en casi todas las variables. En primer lugar, en la comparación de medidas pretest entre grupo experimental y control en lista de espera, no se evidenciaron diferencias significativas en las puntuaciones obtenidas en las variables competencias matemáticas y variables de inteligencia. Esto indica que la distribución de los datos fue inicialmente homogénea en ambos grupos, lo que garantiza control de amenazas a la validez interna (Kazdin y Gutiérrez, 2001).

En cuanto a la efectividad de la intervención, se encontraron diferencias significativas desde las medidas pretest a las postest entre el grupo experimental y el grupo control en lista de espera, evidenciándose mejorías en las diferentes competencias matemáticas y en dos de las tres medidas de inteligencia del grupo experimental.

En particular, se observaron algunas diferencias significativas.

En la variable Sistema Numérico Oral, después del entrenamiento, los niños del grupo experimental lograron una mejoría significativa con respecto al grupo control en lista de espera. Se encontró que los participantes del grupo experimental tuvieron un mayor dominio en la secuencia numérica verbal al desarrollar correctamente tareas de conteo (hasta el número más alto, con límites, dentro de un intervalo, hacia atrás, de diez en diez y de dos en dos). Esto daba cuenta de un mayor nivel de adquisición y elaboración de la cadena numérica, así como una mejor capacidad de atribuir una palabra - número a elementos de un conjunto y una mejora en la comprensión de la tarea de numerar y su cardinalidad (numerar un conjunto de elementos y decir cuántos hay). Esto corrobora estudios realizados por Van Luit, Van de Rijt, Araújo, Aguilar, Aragón, Ruiz, Navarro, Menacho, y 
García-Sedeño (2015) los cuales han diseñado el Test de evaluación de la competencia matemática temprana - informatizado (TEMT-i) y lo han empleado con alumnos con DAM. Uno de los objetivos de su investigación estaba relacionada con la comprensión del sistema numérico y el funcionamiento de algunas estrategias de solución de problemas: reversibilidad $(5 \times 9=9 \times 5)$; asociación $(9 \times 7=$ $10 \times 7-1 \times 7)$, etcétera. En el estudio concluyeron que el entrenamiento en este campo es fundamental para mejorar las DAM en niños en etapas escolares iniciales.

En la variable Operaciones con Enunciado Aritmético, los niños del grupo experimental mostraron mejoría significativa con respecto a los niños del grupo control en lista de espera en cuanto a la ejecución de operaciones sencillas tales como sumas y restas. Esto se logró mediante el entrenamiento a través de objetos concretos y objetos que hacían parte de su cotidianeidad. Se usaron estrategias del tipo: a) dar atributos al número (ej: 3 canicas, 18 panes) y b) vinculando las operaciones a acciones como compra, venta, préstamo y/o reparto. Tal como afirma Vergnaud, (1988) el entrenamiento en este tipo de estrategias permite a los niños realizar las operaciones de forma eficiente porque contribuye a estructurar las diferentes formas aditivas mediante tres elementos: estado inicial, conversión y estado final. Esto fue con el fin de darle sentido a las operaciones aditivas, y no sólo quedándose en la memorización de un algoritmo, sino llevarlo a la resolución de problemas para mejorar los procesos en competencias matemáticas de los niños.

Por otra parte, la variable Operaciones con Enunciado Verbal da cuenta de la resolución de problemas a partir de un enunciado verbal y de las competencias que están en juego (aritméticas, planificación, lectura, representación y comprensión verbal). Los resultados arrojan diferencias significativas entre los niños del grupo experimental y los niños del grupo control en lista de espera. Los del grupo experimental, muestran mejoría significa- tiva en las competencias mencionadas. Estas competencias son necesarias para la comprensión y posterior resolución de problemas, y se observó que lograban solucionar la mayoría de los problemas planteados en la evaluación. Esto, a su vez, se sustenta con lo planteado por Zentall y Ferkis (1993), ya que cuando alguna de estas competencias es pobre, la resolución de problemas se entorpece, derivando así en dificultades en el desarrollo de las competencias generales en matemáticas. Otros autores como Fuchs y Fuchs (2002) corroboran que la comprensión del enunciado es un requisito imprescindible para solucionar problemas matemáticos. Jordan y Hanich, (2000) demuestran que al realizar una prueba con niños con DAM y Dificultades de Aprendizaje en Lectura (DAL) encontraron que presentan más dificultades en solución de problemas que los alumnos con sólo DAM, y concluyen que estas dificultades son aún mayores conforme aumenta la complejidad del texto de los problemas matemáticos.

La variable Conocimientos Conceptuales no arrojó diferencias significativas entre el grupo control y experimental. A pesar de que en el PAMATH-C (Sánchez-Acero y GarcíaMartín, 2019b) existían actividades desde las sesiones 2 a la 5 que entrenaban la competencia de razonamiento verbal-matemático con ejercicios como: Dime algunas reglas o normas que utilizas en el aula de matemáticas. ¿Cuándo se debe utilizar esta regla? ¿Para qué sirve esta regla?, con el fin de conseguir que los niños aprendieran a derivar reglas a partir de su almacén semántico en matemáticas, este entrenamiento no pareció ser suficiente. Una de las razones podría ser que los niños también tenían un nivel de vocabulario medio - bajo que no les permitió beneficiarse más de este entrenamiento. Esto va en consonancia con los estudios que hablan de la importancia de un buen repertorio verbal para que los niños aprendan competencias matemáticas y aprendan a razonar para la extracción de reglas y para la resolución de problemas (Montague y Applegate, 2000). 
En la variable Estimación del Tamaño, también se encontraron diferencias significativas ente el grupo experimental y el grupo control en lista de espera. Los estudiantes del grupo experimental mejoraron su nivel en esta competencia que está relacionada con los conceptos de magnitud y cantidad. Brousseau (1992) indica que la medición implica que la cantidad numérica es aprendida mediante las relaciones grande - pequeño y la asignación de un número a dicha magnitud. Los niños entrenaron esta habilidad en la PAMATH-C (Sánchez-Acero y García-Martín, 2019b), mediante actividades de indicación de dónde está la cantidad más grande, la más pequeña, qué es mayor, qué es menor y qué es igual. La estimación del tamaño está relacionada con la comprensión del sistema numérico, en particular, la compresión del sistema de base 10; los niños mostraron mejoría en la comprensión de la cantidad expresada por los números representados y mejoraron también en la traducción de esa cantidad en el código de salida pertinente (ej: saber que 4 se dice cuatro, y que 84 se dice ochenta y cuatro y no ocho-cuatro). De igual manera, se observó mejoría en la capacidad de captar la representación decimal de los números (por ejemplo: $\$ 20=$ dos monedas de \$10), lo cual se reflejó en una mayor capacidad de contar elementos y grupos, reconocer la igualdad entre diez elementos y una decena. Estos razonamientos son necesarios e importantes para adquirir los sistemas de medida y realizar cálculos (ej: 58 $+14=58+10+4)$ sin necesidad de acudir al conteo de unidades. Esto corrobora investigaciones como la de Ruiz-Ahed (2010) donde enuncia que uno de los elementos importantes para la estimación del tamaño está basado en la comprensión del sistema en base 10 y la magnitud en objetos continuos.

Respecto a la Variable Operaciones Lógicas, también se encontraron diferencias significativas entre el grupo experimental y control en lista de espera. Los niños del grupo experimental mejoraron también significativamente en esta variable. Todo indica, que al haber mejorías, influye en las capacidades lógicas de seriación, operaciones de clasificación y conservación. Es decir, los niños lograron ordenar objetos a partir de sus diferencias (mayor - menor, por ejemplo), agrupar objetos en conjuntos comunes, abstrayendo sus diferencias, basándose únicamente en las características comunes, y admitir que el número de objetos de un conjunto sólo puede cambiar si se modifica la cantidad al agregarle o quitarle objetos. Investigaciones como las de Villalba e Hidalgo (2018) indican el proceso operativo de los niños muestra los diferentes niveles de desarrollo de sus competencias matemáticas, en este caso, el operar de forma eficiente mediante elementos concretos permite mejorar su comprensión al realizar operaciones.

Además, se presentó una mejoría en la composición aditiva, lo que indica que comprenden que un todo puede descomponerse en dos partes, y que éstas hacen parte del mismo conjunto de elementos que resulta ser más grande que las partes incluidas en él. Lo anterior tiene correspondencia con el estudio de Geist (2006) el cual enuncia que el entrenamiento en la seriación y la clasificación en los niños permiten a estos mejorar sus habilidades en la alineación de objetos por orden de tamaño, característica, y que pueden construir torres de bloques, por ejemplo, de distinto tamaño y forma, de la misma manera que pueden establecer diferencias entre "más grande o más pequeño que" y "es de esta clase o no" creando así asociaciones más generales. Sumado a lo anterior, como otro resultado relevante, se encontró que las puntuaciones de inteligencia, concretamente la Velocidad de Procesamiento y Razonamiento Verbal mostraron también un cambio estadísticamente significativo en las puntuaciones postest del grupo experimental. Esto señala que los estudiantes lograron una mayor velocidad para identificar información, manipularla y emitir un resultado, además de mejorar en el nivel de razonamiento concreto y abstracto a nivel matemático. Los resul- 
tados de este estudio confirman los resultados encontrados por otros estudios que hablan de que los entrenamientos que estimulan el razonamiento (sobre todo verbal) y la velocidad para manipular información suelen mejorar las puntuaciones en CI (Cociente Intelectual) medidas a través de tests de inteligencia tradicionales (Desoete y Grégorie, 2006; Ferroni, Barreyro, Mena, y Diuk, 2019).

Por lo tanto, teniendo en cuenta lo anterior, como resultado general de la aplicación del protocolo basado en potencial de aprendizaje contribuye al desarrollo de competencias matemáticas básicas, fortalece la metacognición y tiene un efecto significativo en la disminución de las DAM. Esto se relaciona con los resultados obtenidos en diferentes estudios (Mato-Vázquez, Espiñeira y López, 2017; Mera-Mendes y Peña, 2011; Mevarech y Kramarski, 2017; Moreno y Daza, 2014) donde se menciona que, al trabajar con estas estrategias, los niños con estas dificultades muestran un avance en el desarrollo de las competencias matemáticas básicas que se ve reflejado en su aprendizaje y desempeño en actividades con componente matemático.

Como limitaciones de este estudio, se pueden señalar el tamaño de la muestra y las dificultades logísticas de los centros escolares para realizar las evaluaciones y el entrenamiento. Sería necesario probar este tipo de protocolo de intervención en una muestra más grande y en otras poblaciones colombianas para garantizar estos resultados. Si el entrenamiento se pudiera hacer en jornada extraescolar también se lograría un menor entorpecimiento de las actividades académicas rutinarias de los niños y se podría facilitar un desarrollo fluido.

Es interesante seguir trabajando en la detección de dificultades de aprendizaje en matemáticas en niños de otras edades (más pequeños que la muestra de este estudio). Una identificación temprana puede hacer que, con el acompañamiento/entrenamiento adecuado, el niño no llegue a presentar dificultades mayores ni que estas dificultades se genera- licen a otras áreas del conocimiento. Por otro lado, sería interesante realizar un estudio en profundidad para detectar qué factores (variables) pueden considerarse de riesgo o protectores para el desarrollo, o no, de dificultades en el aprendizaje de las matemáticas.

\section{Referencias bibliográficas}

Aguilar, M., Aragón, E. y Navarro, J. I. (2015). Las dificultades de aprendizaje de las matemáticas (DAM). Estado del arte. Revista de Psicología y Educación, 10(2), 13-42.

American Psychiatric Association [APA]. (2013). Diagnostic and Statistical Manual of Mental Disorders Fifth Edition (DSM 5). Washington D.C.: American Psychiatric Association.

Blanco, M. (1999). Desarrollo de un instrumento de evaluación, diagnóstico y orientación curricular del área de Matemáticas en los primeros años de escolaridad: Prueba evolutivo-curricular de matemáticas de Tordesillas (PRE-CUMAT). Premios nacionales a la innovación educativa 1999. Manuscrito no publicado. Madrid: MEC-CIDE. https://redined. mecd.gob.es/xmlui/handle/11162/83344

Brousseau, G. (1992). El peso de un recipiente. Estudio de los problemas de la medición en CM. Gran N, 50, 65-87.

Butterworth, B. (2005). The development of arithmetical abilities. Journal Child Psychology and Psychiatry, 46, 3-18. https://doi. org/10.1111/j.1469-7610.2004.00374.x

Calero, M. D., Navarro, E., Armedo, M. L., GarcíaBerben, T. M. y Robles, P. (2000). Estimación del potencial de rehabilitación en ancianos con y sin deterioro cognitivo asociado a demencias. Revista Española de Geriatría y Gerontología, 35(2), 44-50.

Calero, M. D. y Marquez, J. (1998). Psychometric properties of a Learning Potential Tests for reading: The Picture Word Game. European Journal of Psychological Assessment, 14(2) 124-133.

Connolly, P. (1998). Keymath revised/Normative update. Circle Pines, MN: America Guidance. 
Desoete, A. y Grégorie, J. (2006) Numerical competence in young children and in children with mathematics learning disabilities. Learning and Individual Differences, 16, 351-367.

Fernández-Ballesteros, R., Espinosa, M. J., Colom, R. y Calero, M. (1997). Contextual and Personal Sources of Individual Differences in Intelligence: Empirical Results. En J. Kingma y W. Tomic (Eds.), Advances in Cognition and Ecucational Practice (pp. 221-274). Londres: Jai Press.

Feuerstein, R. (1984). On the desirability of preserving family and communal traditions. En The integration of immigrant adolescent: A selection of articles of Youth Aliyah. Jerusalem: Jewish Agency.

Feuerstein, R. (1990a). The cognitive modifiability of persons with Down Syndrome. En E. Chigier (Ed.), Looking up at Down Syndrome. Tel Aviv y Londres: Freund.

Feuerstein, R. (1990b). Mediating cognitive processes to the retarded performer. En M. Schwebel, C. Maher y N. Fagley (Eds.), Promoting cognitive growth over the life-span. Hillsdale, NJ: Erlbaum.

Feuerstein, R. (1990c). The theory of structural cognitive modifiability. En B. Presseisen (Ed.), Learning and thinking styles: Classroom interaction. Washington, DC: National Education Association.

Feuerstein, R. (1996). The mediated learning experience: Langeveld memorial lecture. University of Utrecht.

Feuerstein, R. y Rand, Y. (1974). Mediated Learning Experience: An outline of proximal etiology for differential development of cognitive functions. Journal of International Council of Psychology, 9-10, 7-37.

Ferroni, M. V., Barreyro, J. P., Mena, M. y Diuk, B. G. (2019). Perfiles cognitivos de niños de nivel socioeconómico bajo con dificultades en la velocidad lectora: Análisis de los resultados de una intervención. Interdisciplinaria, 36(1), 273-288. https://doi.org/10.16888/ interd.2019.36.1.18.

Fuchs, L. S. y Fuchs, D. (2002). Mathematical problem solving profiles of students with mathematics learning disabilities with and without reading disabilities. Journal of Learning Disabilities, 35, 563-573.

Geary, D. C. (1993). Mathematical disabilities: Cognitive, neuropsychological, and genetic components. Psychological Bulletin, 114, 345-362. https://doi: 1.137/332909.114.2.345.

Geary, D. C. (2004). Mathematics and learning disabilities. Journal of Learning Disabilities, 37, 4-15. https://doi: 10.1177/00222194040370010201

Geary, D. C. (2011). Consequences, characteristics, and causes of mathematical learning disabilities and persistent low achievement in mathematics. Journal of Developmental and Behavioral Pediatrics, 32, 250-263. https:// doi:10.1097/ DBP.0b013e318209edef.

Geary, D. C. y Hoard, M. K. (2001). Numerical and arithmetical de cits in learning-disabled children: Relation to dyscalculia and dyslexia. Aphasiology, 15, 635-647. http://dx.doi. org/10.1080/02687040143000113

Geist, E. (2006). Los niños nacen matemáticos: Animando y promoviendo el desarrollo temprano de los conceptos matemáticos en niños menores de cinco años. Ponencia presentada en el I Congreso Internacional de Lógico-Matemática en educación infantil. (España).

Ginsburg, H. y Baroody, A. J. (1990). Test of Early Mathematics Ability: TEMA-2. Pro-Ed. Madrid: Tea Ediciones.

Grégoire, J., Nöel, M. y Van-Nieuwenhoven, C. (2005). TEDI-MATH; Test para el Diagnóstico de las Competencias Básicas en Matemáticas. Madrid: TEA Ediciones.

Gross-Tsur, V., Manor, O. y Shalev, R. S. (1996). Developmental dyscalculia: Prevalence and demographic features. Developmental Medicine and Child Neurology, 38, 25-33.

Halberda, J., Mazzocco, M. M. y Feigenson, L. (2008). Individual differences in non-verbal number acuity correlate with maths achievement. Nature, 455(7213), 665-668.

Jáuregui, C. A., Mora-Ruiz, C. A., Oviedo-Mosquera, N., Pabón-Rodriguez, Y. L. y Rodriguez-Osorio, A. J. (2016). Manual práctico para niños con dificultades en el aprendizaje: 
enfoque conceptual e instrumentos para su manejo. Bogotá, Colombia: Editorial Médica Panamericana.

Jiménez, G. (2016). How can I help my students with learning disabilities in mathematics? Redimat, 5(1), 56-73. https://doi.org.10.4471/ redimat.2016.1469.

Jordan, N. C. y Hanich, L. B. (2000). Mathematical thinking in second-grade children with differents forms of LD. Journal of Learning Disabilities, 33, 567-578.

Kazdin,A. E. y Gutiérrez, M. G. G. (2001). Métodos de investigación en psicología clínica. México: Pearson Educación.

Lauchlan, F. y Elliot, J. (2001). The Psychological Assessment of Learning Potential. British Journal of Educational Psychology, 71, 647-665.

Mato-Vázquez, D., Espiñeira, E. y López-Chao, V. (2017). Impacto del uso de estrategias metacognitivas en la enseñanza de las matemáticas. Perfiles Educativos, 39(158), 91-111.

Mera-Mendes, D. y Peña, P. (2011). Efectos de la aplicación de estrategias metacognitivas en el rendimiento de los estudiantes de 5to grado al realizar operaciones con números racionales. Revista de Investigación, 35(73), 311-330.

Mevarech, Z. y Kramarski, B. (2017). Matemáticas críticas para las sociedades innovadoras: el papel de las pedagogías metacognitivas. http://dx.doi.org/10.1787/9789264273078-es

Moll, K, Göbel, S., Gooch, D., Landerl, L., Karin, S. y Margaret. T. (2014). Cognitive Risk Factors for Specific Learning Disorder. Journal of Learning Disabilities. https://doi. org.10.1177/0022219414547221.

Montague, M. y Applegate, B. (2000). Middle school students' perceptions, persistence, and performance in mathematical problem solving. Learning Disability Quarterly, 23, 215-227.

Moreno, A. y Daza, B. (2014). Incidencia de las estrategias metacognitivas en la resolución de problemas en el área de matemáticas. (Tesis de Maestría). Universidad Javeriana, Bogotá, Colombia.

Mutlu, Y. y Akgün, L. (2017). The effects of computer assisted materials on approximate number skills of students with dyscalculia. Tojet: The Turkish Online Journal of Educational Technology, 16(2), 119-136.

Olea, R., Ahumada, H. y Líbano, L. H. (1993). PCM, Prueba de Comportamiento Matemático. Centro de Perfeccionamiento, Experimentación e Investigaciones Pedagógicas. Santiago de Chile. Chile. http://elpsicoasesor. com/pcm-prueba-de-comportamiento-matematico-ricardo-olea/

Olkun, S., Altun, A., Cangöz, B., Gelbal, S. y Sucuoğlu, B. (2012). Developing Tasks for Screening Dyscalculia Tendencies. Berlin: E-Leader.

Robles, A. y Calero, M.D. (2003). Evaluación del potencial de aprendizaje en niños con síndrome down. Siglo Cero, 34(2), 14-25.

Romero, J. y Lavigne, R. (2004). Dificultades en el aprendizaje: Unificación de criterios diagnósticos. Consejería de educación. [Versión Adobe Digital]. http://www. uma. es/media/ files/LIBRO_I. pdf.

Romero, J. y Lavigne, R. (2011). Dificultades en el Aprendizaje: Unificación de criterios. Consejería de Educación. Sevilla: Junta de Andalucía Editores.

Ruiz-Ahed, Y. M. (2010). Dificultades de aprendizaje de las Matemáticas. Temas para la Educación. Andalucia: Federación de Enseñanza de CC.OO.

Sánchez-Acero, A. y García-Martín, M. B. (2019a). Programa de Potencial de Aprendizaje para el entrenamiento en DAM [PAMATH-C]. (Tesis Doctoral). Fundación Universitaria Konrad Lorenz, Bogotá, Colombia.

Sánchez-Acero, A. y García-Martín, M. B. (2019b). PAMATH-C potencial de aprendizaje en matemáticas: Programa de entrenamiento para niños. En Matemática: Ciência e Aplicações (pp. 226-240). https://doi.org.10.22533/ at.ed.86719071025

Sternberg, R. y Grigorenko, E. L. (2001). All testing is dynamic testing. Issues in Education, $7(2)$.

Van Luit, E. H. J., Van de Rijt, B., Araújo, A., Aguilar, M., Aragón, E., Ruiz, G., Navarro, J. I., 
Menacho, I. y García-Sedeño, M. (2015). Test de evaluación de la competencia matemática temprana-informatizado (TEMT-i). Madrid: EOS.

Vergnaud, G. (1988). Multiplicative structures. En J. Hiebert y M. Behr, Number Concepts and Operations in the Middle Grades (pp. 141-161). Reston, Virginia: The National Council of Teachers of Mathematics, Inc.

Villalba Garcés, J. y Frisancho Hidalgo, S. (2018). Evaluación de operaciones lógico-matemáticas mediante dos métodos distintos en niños del pueblo indígena Shipibo-Konibo. Interdisciplinaria, 35(1), 217-238. https://doi. org/10.16888/interd.2018.35.1.12

Wechsler, D., Kaplan, E., Fein, D., Kramer, J., Morris, R., Delis, D. y Maerlender, A. (2004). Wechsler Intelligence Scale for Children -
Fourth edition integrated. San Antonio, TX: Harcourt Assessment.

Wiedl, K. H., Wienöbst, J. y Schöttke, H. (2000). Estimating rehabilitation potential in schizophrenic subjects. En H. D. Brenner, W. Böker y R. Genner (Eds.), The treatment of schizophrenia: status and emerging trends (pp. 145-167). Seatle: Hogrefe and Hubers Publishers.

Zentall, S. S. y Ferkis, M. A. (1993). Mathematical problem-solving for ADHD children with and without learning disabilities. Learning Disability Quarterly, 16(1), 6-18.

Zerafa, E. (2015). Helping children with dyscalculia: a teaching programme with three primacy school children. Procedia social and behavioral sciences, 191(2), 1178-1182. https://doi. org/10.1016/j.sbspro.2015.04.516.

Recibido: 5 de junio de 2019 Aceptado: 5 de noviembre de 2020 